A N N A L E S Annales de Bretagne et des Pays de l'Ouest

\title{
Philippe DEPREUX, Les sociétés occidentales du milieu du VIe à la fin du IXe siècle,
}

Jean-Pierre Delumeau

\section{(2) OpenEdition \\ 1 Journals}

Édition électronique

URL : https://journals.openedition.org/abpo/1433

DOI : $10.4000 /$ abpo. 1433

ISBN : 978-2-7535-1490-4

ISSN : 2108-6443

Éditeur

Presses universitaires de Rennes

Édition imprimée

Date de publication : 20 juillet 2003

Pagination : 239-241

ISBN : 978-2-86847-874-0

ISSN : 0399-0826

\section{Référence électronique}

Jean-Pierre Delumeau, « Philippe DEPREUX, Les sociétés occidentales du milieu du Vle à la fin du IXe siècle, ", Annales de Bretagne et des Pays de l'Ouest [En ligne], 110-2 | 2003, mis en ligne le 20 juillet 2005, consulté le 22 juillet 2022. URL : http://journals.openedition.org/abpo/1433 ; DOI : https:// doi.org/10.4000/abpo.1433 


\section{Comptes rendus}

DepreuX, Philippe, Les sociétés occidentales du milieu du Ve à la fin du IXe siècle, Rennes, PUR, " Histoire ", 2002, 304 p.

Les programmes de concours donnent naissance à une pléiade de livres, dont les titres reprennent souvent l'intitulé de la question elle-même. Beaucoup ne prétendent qu'à un usage $a d h o c$, mais même dans cette perspective, certains peuvent constituer des outils de travail plus durables : ainsi, l'ouvrage collectif publié par les éditions Atlande, qui, à l'instar de ses prédécesseurs élaborés sous l'efficace direction d'Isabelle Heulant-Donat, se présente comme un recueil de "fiches " denses et actualisées, regroupées en grands ensembles. Toutefois, le livre de Philippe Depreux, que publient les PUR, tranche sensiblement sur le reste de la production. Le propos de ce volume de 300 pages se veut "modeste" (p. 3) : suggérer une grille de lecture (parmi d'autres possibles...), présenter quelques exemples, offrir des " pistes de réflexion " aux étudiants préparant les concours. Or, disons d'emblée que ce livre va très audelà et fournit une synthèse, d'une rare qualité à bien des égards sur les sociétés occidentales du milieu du vi siècle à la fin du $\mathrm{IX}^{\mathrm{e}}$ siècle. Toutefois, par la densité et la diversité de l'information, il se situe très en avant du niveau usuel des étudiants de concours; et il ne peut être lu avec profit, et avec un réel plaisir, que par des lecteurs déjà familiarisés avec les sociétés et les cadres historiques du haut Moyen Âge.

Le livre de Philippe Depreux s'appuie sur une information remarquable dont témoigne la bibliographie. Qualifiée de " sommaire " par l'auteur, celle-ci est loin de recenser tous les ouvrages consultés; elle comporte pourtant quelque 600 titres, dont une forte proportion en langues étrangères, en allemand tout particulièrement (plus encore qu'en anglais). Ph. Depreux nous transmet donc, sur des aspects cruciaux de la question, l'apport actualisé de la bibliographie allemande; on ne s'étonnera donc pas que l'exposé soit très riche et nuancé sur le monde germanique au sens large. Les Îles britanniques sont elles aussi fort bien éclairées : non seulement l'Angleterre anglo-saxonne, mais aussi l'Irlande, sur laquelle l'auteur nous offre un corpus de développements remarquables, bien que dispersés, et difficilement accessibles ailleurs. Les mondes plus méridionaux - Italie, péninsule Ibérique - seraient, comparativement, plus légèrement représentés en appendice : la notice du plaid réuni en 804 en Istrie par des missi de Charlemagne, commentée par exemple dans le chapitre vIII, "Pouvoir et autorité" (p. 221-224).

Le plan de l'ouvrage peut paraître déroutant, tant par sa composition d'ensemble que dans le détail. Cela résulte de la difficulté à trouver des angles d'approche adéquats sur une question aussi vaste et diverse. En introduction (p. 12), l'auteur explicite la démarche qui est la sienne : "Ce livre est conçu comme un 
diptyque dont le gond serait constitué du chapitre médiant (v) qui traite de la question centrale de la place de l'individu dans la société. Les quatre premiers chapitres visent à cerner les contextes (spaciaux, sociaux, mentaux) dans lesquels les hommes et les femmes du haut Moyen Âge évoluaient, alors que les derniers sont destinés à présenter brièvement les tendances récentes de l'historiographie et les principaux thèmes dont le traitement constitue un enjeu pour la compréhension des règles de la vie sociale. " Ce qui rend compte de la brièveté, souvent réelle, de l'évocation de certains points dans ces derniers chapitres : on peut ici recourir à des livres accessibles. Mais faisons aussi observer que la problématique très subtile retenue pour la définition de des thèmes présentés, qui bouleverse les thématiques " scolaires usuelles ", rend la chose plus complexe que l'énonce l'auteur. Le chapitre III, ("Guerriers et paysans ") est une synthèse judicieuse, qui, entre autres, pose bien le problème de la limite entre paysannerie et notables locaux, mais brève (p. 105-114). À l'inverse, le chapitre VII ("Culture et communication ") est à bien des égards un des points forts du livre.

Les renouvellements apportés par l'historiographie récente, de même que le souci pédagogique, conduisaient l'auteur à dépasser, même formellement, les présentations usuelles. Le chapitre I vise à " Appréhender les sociétés du haut Moyen Âge ". Or l'incontournable exposé sur les sources (typologie, méthodes d'analyse), au demeurant remarquable (ainsi, sur la législation), se retrouve reporté en fin de chapitre, l'auteur ayant estimé que le lecteur devait être au préalable averti de la problématique d'ensemble. Relevons ici le sous-titre crucial de la page 22 : " Aux environs de 700, une ligne de partage entre les eaux? ", ce à quoi $\mathrm{Ph}$. Depreux répond implicitement - et avec raison - par l'affirmative. Essentiel est aussi le point consacré au Christianisme comme facteur structurant de la société : mais ce cadre chrétien est, au fil de l'ouvrage, presque partout présent. Non moins fondamental est ensuite le chap. II, "Les conditions de vie " : le milieu et ses aléas (famines, maladies : peut-être eussé-je davantage insisté sur la gravité des effets démographiques de la peste, présente jusqu'au milieu du VIII ${ }^{\mathrm{e}}$ siècle); l'insertion des individus et des groupes humains dans ce milieu et les rapports de l'homme avec l'espace. Dans un exposé nourri par une confrontation constante avec les rapports de l'archéologie et les relectures récentes des sources écrites, et par un dialogue suivi avec une ample bibliographie, on trouve d'excellents développements sur le peuplement et les formes d'occupation du sol, et aussi sur les communications, les marchés, ou la frontière.

L'auteur a signalé la place centrale du chapitre v, "L'individu dans le groupe ». En fait, les chapitres IV (" Des groupes sociaux privilégiés "), v et VI ("Alliances et solidarités ") constituent un ensemble, sur un volet fondamental et renouvelé par l'historiographie récente : on pense évidemment à la thèse de Régine Le Jan mais Philippe Depreux emprunte également beaucoup à l'historiographie allemande. L'approche retenue est ici plus proche de celle de R. Le Jan que de celle de K. F. Werner. On trouvera dans le chap. IV des éléments actualisés sur des aspects aussi cruciaux que les critères de la noblesse, l' "aristocratie d'Empire " aux temps carolingiens, l'entourage royal, les liens entre aristocratie et monastères, et aussi, p. 138, sur les réseaux de prière, récemment mis en lumière. Heureusement introduits par un, passage de la vie de S. Wulfran (présenté par S. Lebecq) sur le baptême manqué du chef frison Radbod, le chap. v intègre les rapports récents, et substanciels, de l'onomastique, de l'anthropologie de la famille, voire de la gender history.

À partir des derniers points de ce chapitre v, on entre effectivement dans des aspects plus anciennement défrichés, et les points abordés sont plus succincts, 
comme l'auteur lui-même nous en avertissait en introduction. L'exposé reste très pertinent, mais après les sommets des précédents chapitres, on souhaiterait, par gourmandise, plus d'approfondissements. La sensibilité de Ph. Depreux aux aspects culturels nous y ramène au chapitre VII ("Culture et communication "), notamment sur les fêtes et spectacles, les chants et épopées, les langues et les traductions. Enfin, les chapitres VIII ("Pouvoir et autorité ") et Ix ("Ordre et désordre ") constituent un dernier ensemble, d'orientation plus " politique ".

Une brève recension ne permet certes pas de rendre compte de la substance d'un livre aussi riche qui, redisons-le, demande au préalable une connaissance déjà approfondie et diversifiée du haut Moyen Âge : l'exposé ne cesse de naviguer d'un bout à l'autre du cadre historique et géographique. De ce fait, le lecteur même initié regrettera de n'être pas plus aidé dans sa lecture, et le manque d'un index se fait cruellement sentir. D'autant que la thématique adoptée, volontairement innovante, amène des regroupements déroutants et des chapitres malgré tout hétérogènes. La sépulture des défunts s'intègre malaisément dans un chapitre II consacré aux conditions de vie; à l'inverse, l'exposé sur les marchands est éclaté entre le chapitre II et la fin du chapitre IV. Plus ponctuellement, l'étudiant peu familiarisé avec le vieil anglais pourrait souhaiter en note une traduction littérale des vers fort judicieusement insérés page 159.

De fait, le lecteur a d'emblée le sentiment d'avoir entre les mains non pas un manuel, mais une synthèse de haute qualité; et lorsqu'il se voit, par moments, ramené aux perspectives plus utilitaires des concours, il se prend à regretter que ce livre déjà très dense ne fût deux fois plus gros. Mais tel qu'il se présente, le livre de Philippe Depreux, brillant, foisonnant, élitiste aussi, constitue sur la question le livre du maître, plutôt que celui du néophyte. Il constitue aussi une synthèse actualisée, appelée à prendre une place durable parmi les ouvrages de référence sur le haut Moyen Âge.

Jean-Pierre DeLUMEAU

RAYNAUD, Christiane, "À la hache », histoire et symbolique de la hache dans la France médiévale (XIII'-XVe siècle), Paris, Le Léopard d'or, 2002, 700 p.

Christiane Raynaud, bien connue par différents travaux, dont La violence au Moyen Âge (Paris, 1990) et le très précieux Commentaire de document figuré en histoire médiévale (Paris, 1997), s'est attaquée à cet objet central que fut la hache dans la praxis et dans l'imaginaire des Français entre 1200 et 1500. Elle a mené à bien son entreprise avec l'ardeur d'un moine défricheur de la haute époque et avec l'abnégation d'un convers cistercien. Rude tâche que de recenser les différents usages, les mille et une utilisations, les innombrables occurences et les multiples sens de la bonne vieille cognée, chère aux manants, aux défricheurs, aux jouteurs et aux combattants, dont le Grand Ferré et Du Guesclin! On ne pouvait rêver prolongement plus logique à La violence au Moyen Âge et mise en œuvre plus judicieuse des conseils prodigués dans le manuel précité, que la mise sur le pavois de cet outil précieux entre tous, susceptible de se transformer, le cas échéant, en une arme des plus redoutables. Au service de cette entreprise ambitieuse, qui se donne pour horizon ultime d'essayer d'évaluer le nombre de haches " civiles " et " militaires " en usage dans la France des Valois, Christiane Raynaud déploie une érudition stupéfiante, pour ne pas dire confondante. À preuves, la copieuse liste des manuscrits consultés en France et à 